\title{
Recognition of Affective States in Virtual Rehabilitation using a Late Fusion with Semi-Naïve Bayesian Classifier
}

\author{
blinded \\ blinded@blinded \\ blinded \\ blinded, blinded
}

\author{
blinded \\ blinded@blinded \\ blinded \\ blinded, blinded
}

\author{
blinded \\ blinded@blinded \\ blinded \\ blinded, blinded
}

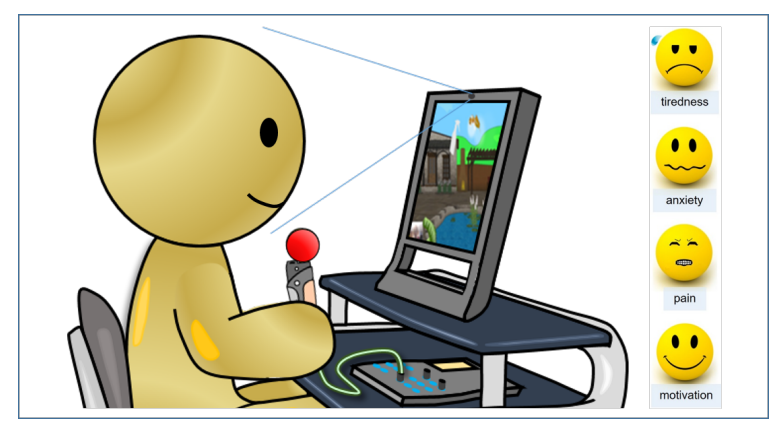

Figure 1: Affective states recognition system that includes fingers' pressure, hand movements, and facial expressions, using a late fusion with Semi-Naïve Bayesian classifier, in a virtual rehabilitation platform.

\begin{abstract}
Virtual rehabilitation platforms may tailor the rehabilitation tasks to the patients' needs if they could recognize the patient's affective state. Affective states recognition systems can enhance their performance if they receive data coming from different sensors of human behaviour. In this work, we propose a late Fusion using Semi-Naïve Bayesian classifier (FSNB) as a multimodal affective states recognition system to infer four states: tiredness, anxiety, pain, and motivation, from observable metrics of hand movements, fingers' pressure and facial expressions of post-stroke patients. Data streams were recorded from 5 post-stroke patients while they attended virtual rehabilitation therapies along 10 sessions over 4 weeks, manifesting the aforementioned states spontaneously. Recognition rates of the FSNB classifier were over $90 \%$ (with a standard deviation of around \pm 0.06 ) of AUC for the four states. These results represent contributions for enhancing the development of affective states recognition systems in virtual rehabilitation.
\end{abstract}

\section{CCS CONCEPTS}

- Human-centered computing $\rightarrow$ Gestural input; • Computing methodologies $\rightarrow$ Supervised learning by classification; Mixture models.

Permission to make digital or hard copies of all or part of this work for personal or classroom use is granted without fee provided that copies are not made or distributed for profit or commercial advantage and that copies bear this notice and the full citation on the first page. Copyrights for components of this work owned by others than the author(s) must be honored. Abstracting with credit is permitted. To copy otherwise, or republish, to post on servers or to redistribute to lists, requires prior specific permission and/or a fee. Request permissions from permissions@acm.org.

PervasiveHealth 2019, May 20-23, 2019, Trento, Italy

(c) 2019 Copyright held by the owner/author(s). Publication rights licensed to ACM.

ACM ISBN 978-x-xxxx-xxxx-x/YY/MM...\$15.00

https://doi.org/10.1145/nnnnnnn.nnnnnnn

\section{KEYWORDS}

affective states recognition, multimodal model, virtual rehabilitation, fingers' pressure, hand movements, facial expressions

ACM Reference Format:

blinded, blinded, and blinded. 2019. Recognition of Affective States in Virtual Rehabilitation using a Late Fusion with Semi-Naïve Bayesian Classifier. In PervasiveHealth 2019: EAI International Conference on Pervasive Computing Technologies for Healthcare, May 20-23, 2019, Trento, Italy. ACM, New York, NY, USA, 6 pages. https://doi.org/10.1145/nnnnnnn.nnnnnnn

\section{INTRODUCTION}

The development of virtual rehabilitation (VR) systems with intelligent interfaces should allow the adaptation of the computational platforms to the particular needs of the patients, including the observation of their affective states. Virtual rehabilitation sessions can be more empathic and motivating if the system is able to consider the affective, physical and psychological states of the patients. Computational models for detecting affective states benefit from including information from different sensors to improve classification rates $[5,14]$. Most of the research mainly include three kind of modalities: visual (facial expressions), audio (vocalization), and text information (written communication) [14]. About $10 \%$ of the research has addressed the use of physiological signals and body movements as other kinds of modalities [5].

Our proposal includes three sensors, one visual (facial expressions) and two related to body movements (hand movements and fingers' pressure). The information is acquired through not invasive sensors, and they can be used in naturalistic applications, for example in rehabilitation platforms at home. 
The integration of these three sensors in a multimodal affective states recognition system that is incorporated in a virtual rehabilitation (VR) platform could accomplish better adaptation to the patients' individual needs.

We also propose a computational model, and we called it Fusion using Semi-Naïve Bayesian classifier (FSNB) to fuse the information of the different sensors. The base classifier for processing the information of each sensor is the Multiresolution Semi-Naïve Bayesian classifier (MSNB) proposed in [15] but a change was made in the late fusion module (changing the majority vote by a Semi-Naïve Bayesian classifier (SNB)), and the new version of the classifier is referred to as MSNB2.

To test the hypothesis that a multimodal affective states recognition system which incorporates a finger's pressure sensor (PRE), a hand movement sensor (MOV) and a facial expressions sensor (FAE) could reach high recognition rates, we used a database of post-stroke patients which includes the records of these three sensors and the identification of four states (affective, physical or psychological): tiredness, anxiety, pain, and motivation [16]. These states were mani-fested spontaneously by the patients whilst they attended their therapies using the virtual rehabilitation system Gesture Therapy, longitudinally over a period of 4 weeks.

The proposed multimodal affective states recognition system represents a contribution to the affective computing field and to the virtual rehabilitation area. The new model could favour intelligent and empathic human computer interactions that address the automatic affective states recognition in everyday life naturalistic virtual rehabilitation systems.

The main contributions of this paper are two: a) A multimodal scheme which includes fingers' pressure, hand movements and facial expressions of post-stroke patients, and b) A late fusion using a SNB classifier which exploits structural improvement of the model.

\section{BACKGROUND}

Different sensors can register the information of several signals to characterize distinct aspects for recognizing affective states from human behaviour. The information of these signals can be combined to explore if the complementation may promisingly enhance the recognition performance [5]. Around $10 \%$ of affective computing research has addressed the integration of modalities related to body movements and other modalities as facial expressions [5, 14] Filntisis et al. (2019) [7] studied affect recognition in child robot interaction, proposing a system which received body expressions and facial expressions information. They used Deep Neural Networks (DNN) for processing each modality (body and facial), and made a late fusion through a fully connected layer. Balomenos et al. (2004) [1] inferred emotional states analyzing the facial expression and the hand gestures. The computational models included fuzzy logic and Hidden Markov Models (HMM) for studying the facial expressions and the hand gestures respectively. The results of each modality (facial and hand) were combined at late fusion using a weighted sum of the two modalities. Gunes and Piccardi $[8,9]$ also investigated on combining facial expressions and hand gestures.

To our knowledge, we are the first proposing the study of combining hand movements and fingers' pressure with facial expressions. For rehabilitation therapies of post-stroke patients, the hand movements and fingers' pressure recovery of an impaired upper limb is a specific target to achieve [20], so it is relevant to include these modalities in a virtual rehabilitation system [3, 10, 19, 20]; even more if we want to incorporate the automatic recognition of the patients' affective states to the virtual rehabilitation platform $[16,17]$.

\section{GESTURE THERAPY PLATFORM}

Gesture Therapy (GT) is a virtual rehabilitation platform for assisting post-stroke patients in their upper limbs rehabilitation exercises (Fig. 2). Patients perform the exercises through serious games.

GT consists of five interacting modules [20]: (a) Physical System (the hardware components) which is compound of a computer, a webcam, and a gripper that the patient holds with the impaired hand; (b) Tracking System which is the software for tracking the gripper's colour ball and for registering the fingers' pressure exerted on the pressure sensor in the front of the gripper; (c) Simulated Environment System where the serious games are presented, and the interaction with the patient is controlled; (d) Trunk Compensation Detector System which is responsible to indicate whether the patient makes compensation; and (e) Adaptation System which is capable of adjusting the games difficulty dynamically, considering patient's performance and where the recognition of affective states will be useful. Some advantages of the GT platform are its portability for being used at home, and its low cost in comparison with robotic systems.

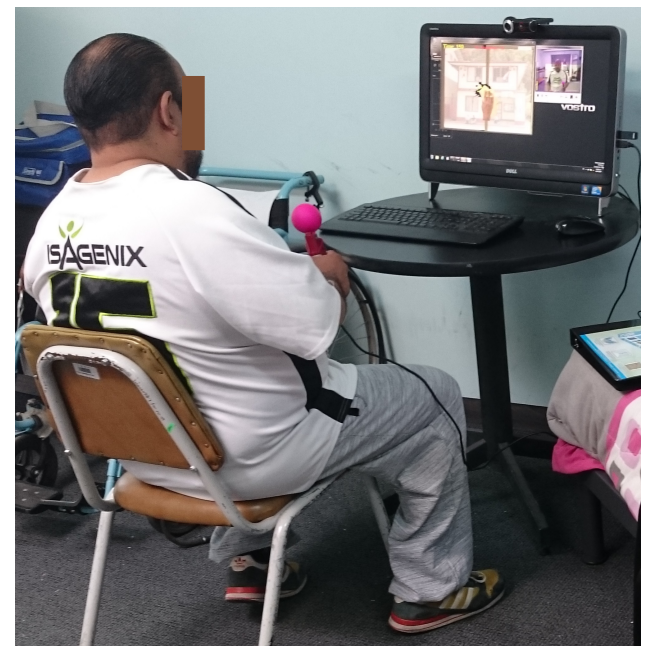

Figure 2: A patient using Gesture Therapy during a rehabilitation session.

\section{DATASET}

An existing dataset of post-stroke patients was used to assess the proposed multimodal system [16]. The dataset contains the tracking of the rehabilitation sessions of 5 post-stroke patients that underwent therapies to recover the mobility of an upper limb, using the GT platform. Frontal videos of the patients were recorded during the rehabilitation sessions. These videos included the face expressions, the hand movements, and the postures of the upper torso. 
The videos were used for psychiatrists to label the patient's states (affective, physical or psychological). A sample was generated for consecutive frames in the considered windows sizes for the multiresolution scheme (see subsection 5.2), containing features for fingers' pressure, features for hand movements, and labels for 4 states: tiredness, anxiety, pain, and motivation. Data in each sample were synchronized through the associated consecutive frames. Each sample of the dataset has 3 features of fingers' pressure (from PRE sensor): (averages of) pressure (Pres), pressure speed (PresSpe) and pressure acceleration (PresAce); 5 features of hand movement (from MOV sensor): (averages of) speed (Spe), acceleration (Ace) and differential location by the axes: $x$ (DifL $x), y$ (DifLy), $z$ (DifLz), and 4 binary labels (of the set $\{-1,1\}$ ), one for each state, indicating presence (1) or absence $(-1)$ of the state.

Patients' facial expressions features were added to the poststroke patients' dataset. An automatic facial expressions recognition system [2] extracted 20 features from each frame of the patients' frontal video. These features (from FAcial Expression sensor: FAE sensor) are distances or angles of geometrical figures over the eyebrow, the eyes and the mouth [18] (Fig. 3). The patients' facial expressions are spontaneous and they expressed complex states: tiredness, anxiety, pain, and motivation. The tags for the states (classes) to each sample were obtained from the consecutive labels assigned by the raters to the video frames. For multimodal classification purposes, the fingers' pressure value, the hand location point of each frame, and the states were synchronized with facial expressions samples because they were obtained by frame.

\section{COMPUTATIONAL MODELS: SNB, MSNB2 AND FSNB}

\subsection{SNB}

Semi-Naïve Bayesian (SNB) classifier is based on Naïve Bayes (NB) classifier [6,13]. Given a sample $s_{a}=\left(a_{1}, a_{2}, \cdots, a_{n}\right)$, and given $A_{i}$ which represents the $i t h$ attribute, the decision rule of NB for a two class problem (the class variable $C$ takes values in $\{-1,1\}$ ), is expressed as:

$$
\operatorname{class}\left(s_{a}\right)=\underset{c \in\{-1,1\}}{\arg \max }\left(\operatorname{Prob}(C=c) \prod_{i=1}^{n} \operatorname{Prob}\left(A_{i}=a_{i} \mid C=c\right)\right)
$$

The multiplication in (1) is support on the naïve assumption that all attributes $A_{i}$ are independent given the class $C$ [13]. To address a more realistic and generic situation, the SNB classifier executes a structural improvement [11-13] to remove and/or to join attributes. The structural improvement (Fig. 4), is based on mutual information and conditional mutual information calculations [4]. After each structural modification is made (elimination or join), the new structure is tested to determine whether classification accuracy is improved. The process is repeated until all attributes have been checked.

\subsection{MSNB2}

Multiresolution Semi-Naïve Bayesian classifier (MSNB) is a binary classifier to explore the occurrence of affective states of interest in the trace over time [15]. The classifier operationalizes several oddsize windows (starting from 3 ) concentric to a current point that

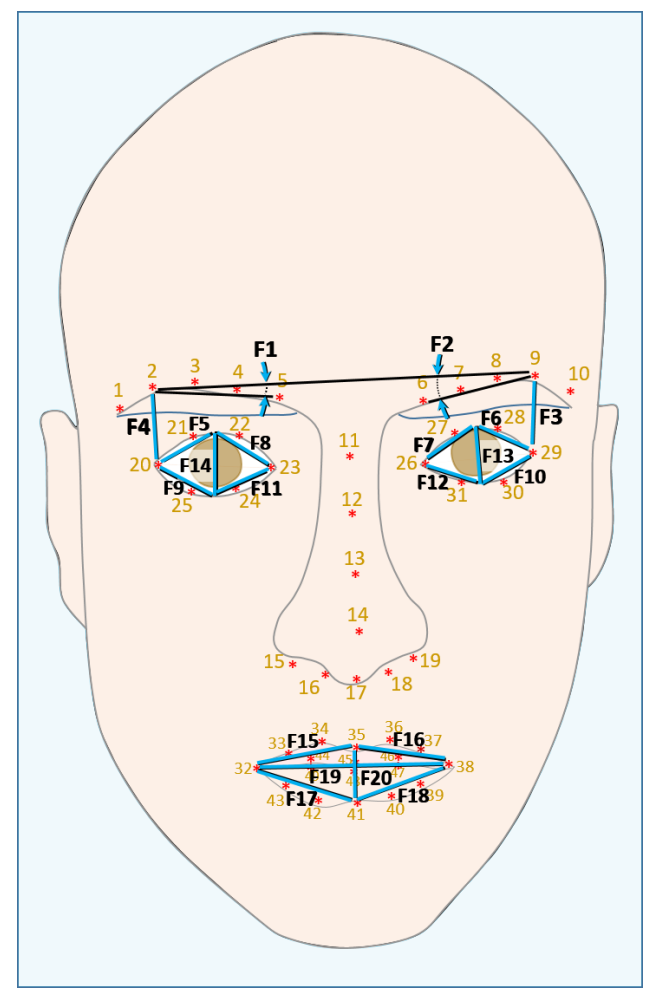

Figure 3: Facial Expressions Features: $F_{i}, i \in\{1,2, \cdots, 20\}$. These features are distances or angles of geometrical figures over the eyebrow, the eyes, and the mouth.

shift simultaneously over the trace, and which it becomes possible to calculate, in the current point environment, several features that may help to discriminate the presence of the affective state (Fig. 5). The name of this classifier has the prefix multiresolution because the windows represent several simultaneous resolutions at the current point of the trace. The classifier represents an ensemble of Semi-Naïve Bayesian classifiers (SNB) with a late (decision level) fusion process by majority vote. Each SNB receives the features coming from a different window size and infers the presence or not of the affective state of interest. At the end, in the fusion stage, the presence or not is decided, by means of the majority vote of the SNBs. Afterward, the majority voting was replaced with a SNB classifier at MSNB late fusion module, and the resulting classifier was called MSNB2. In part a) of the Fig. 6 the architecture of MSNB2 is shown.

\subsection{FSNB}

A MSNB2 classifier is used separately to process the information of each sensor to determine the occurrence or not of a state. Then the late fusion using SNB classifier (FSNB) was proposed as an alternative to fuse the information of state recognition from the different sensors (Fig. 6, part b)). The recognition results of each sensor (PRE, MOV, and FAE) are received at the fusion module and a SNB classifier treats each of them as an attribute and infers the final answer about the presence or absence of the state in consideration. 


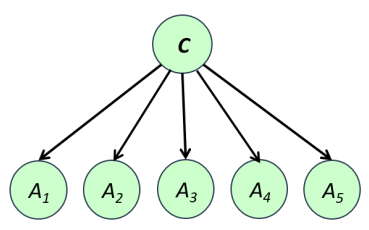

(a) Initial structure

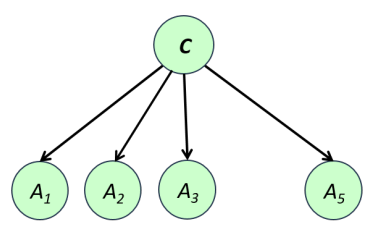

(b) Elimination of $A_{4}$

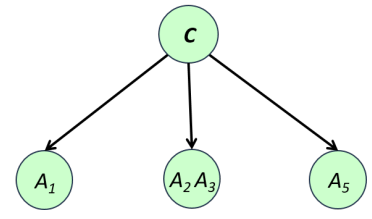

(c) Joining $A_{2}$ and $A_{3}$

Figure 4: Example of the process of structural improvement to obtain a Semi-Naïve Bayesian (SNB) model: (a) An original Naïve Bayes model with 5 attributes (all of them are assumed independent), (b) Attribute $A_{4}$ is eliminated because the mutual information value between $A_{4}$ and the class $C$ is close to zero, (c) Attributes $A_{2}$ and $A_{3}$ are joined into one, as they are considered dependent base upon the mutual information value between $A_{2}$ and $A_{3}$ given the class $C$.

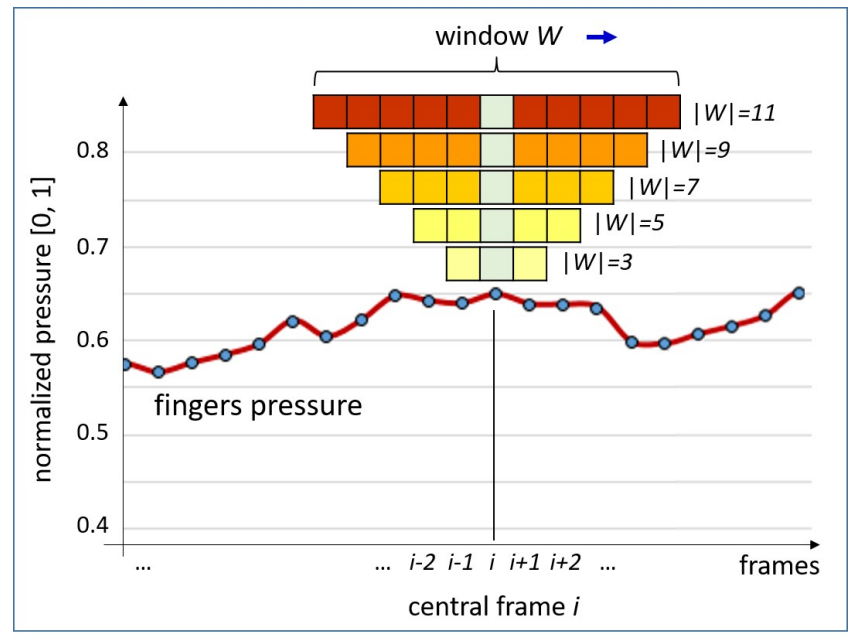

Figure 5: Multiresolution process using several odd-size windows (starting from 3 ) concentric to a current point that shift simultaneously over the trace.

\section{EXTRACTION OF FACIAL EXPRESSIONS FEATURES FOR THE MULTIRESOLUTION SCHEME}

The 20 facial attributes over time for consecutive frames of the patient's frontal video, produced 20 parallel time series. Each of these 20 time series were processed concurrently using the multiresolution scheme (similar as explained at Fig. 5). Therefore, multiresolution feature vectors were obtained by each odd window size and these vectors contained the average of the 20 values within the respective window size.

\section{EXPERIMENTS AND RESULTS}

MSNB2 and FSNB models were independently trained for each state and for each patient, so we had as many FSNB models as (number of states) $\times$ (number of patients), and as many MSNB2 models as (number of states) $\times$ (number of patients) $\times$ (number of sensors). Internal validity of the MSNB2 and FSNB models was established using the stratified 10 -fold cross validation mechanism over all the rehabilitation sessions. Three metrics: accuracy, F-measure, and ROC AUC ${ }^{1}$ were used to assess the results of each classification model and to compare between them.

An experiment was carried out for comparing the performance of MSNB2 for each sensor (PRE, MOV, and FAE) at each state and the performance of FSNB for each state, and over the average performance across the four states.

Facial expressions features for MSNB2 classifier consisted in the average of the 20 facial expressions features of consecutive video frames (in which the face was successfully detected) between the odd window sizes of the multiresolution scheme. Therefore, we used an approach of employing the average trend of the facial expressions features as the elements of the multiresolution vector samples. These vector samples and its corresponding 4 dominant classes tags between the odd windows sizes were the training data to develop the MSNB2 models.

The proposed multimodal affective states recognition system was evaluated to determine whether the three sensors could contribute to increase the recognition scores. Consequently, MSNB2 models were trained for each sensor: PRE, MOV, and FAE, for each patient and each state. Then, we used FSNB for fusing the sensors information. The number of samples was reduced since the face was not detected and was not tracked for several frames in patients' frontal videos. This situation is relevant since it is an example of a failure of one sensor in naturalistic everyday affective states automatic systems. AUC average results across the 10 folds and across the five patients for each specific state (tiredness, anxiety, pain, and motivation) are summarized in Table 1. Patients P2 and P3 are not included in Pain results because they had not samples for pain. According to these results, each sensor contributed to improve classification rates in the multimodal system FSNB, and FAE sensor had the best results among the three sensors.

In Fig. 7, the average results of accuracy, F-Measure and AUC across the 10 folds, the five patients and the four states are plotted. In increasing order for the three metrics, the results are similar: first PRE sensor results, second MOV sensor results, followed by FAE sensor results, and the best results are for the fusion scheme FSNB. FAE results are close to the FSNB results, but the results of all the sensors are almost 80 percent of success.

\section{DISCUSSION}

The proposed multimodal computational model FSNB got results over $93 \%$ with a standard deviation of around \pm 0.06 in the AUC for all the states. Each sensor contributed with results over 70 $\%$ with a standard deviation of around \pm 0.1 in the AUC for all

\footnotetext{
${ }^{1} \mathrm{TP}$ : true positive, TN: true negative, FP: false positive and FN: false negative; accuracy $=(\mathrm{TP}+\mathrm{TN}) /(\mathrm{TP}+\mathrm{FP}+\mathrm{TN}+\mathrm{FN})$; sensitivity $=\mathrm{TP} /(\mathrm{TP}+\mathrm{FN})$; specificity $=\mathrm{TN} /(\mathrm{TN}+\mathrm{FP})$; Precision $=\mathrm{TP} /(\mathrm{TP}+\mathrm{FP}) ; \mathrm{F}-$ measure $=2\left(\right.$ precision ${ }^{*}$ sensitivity $) /($ precision + sensitivity $)$; ROC AUC $=($ Sensitivity + Specificity $) / 2$.
} 


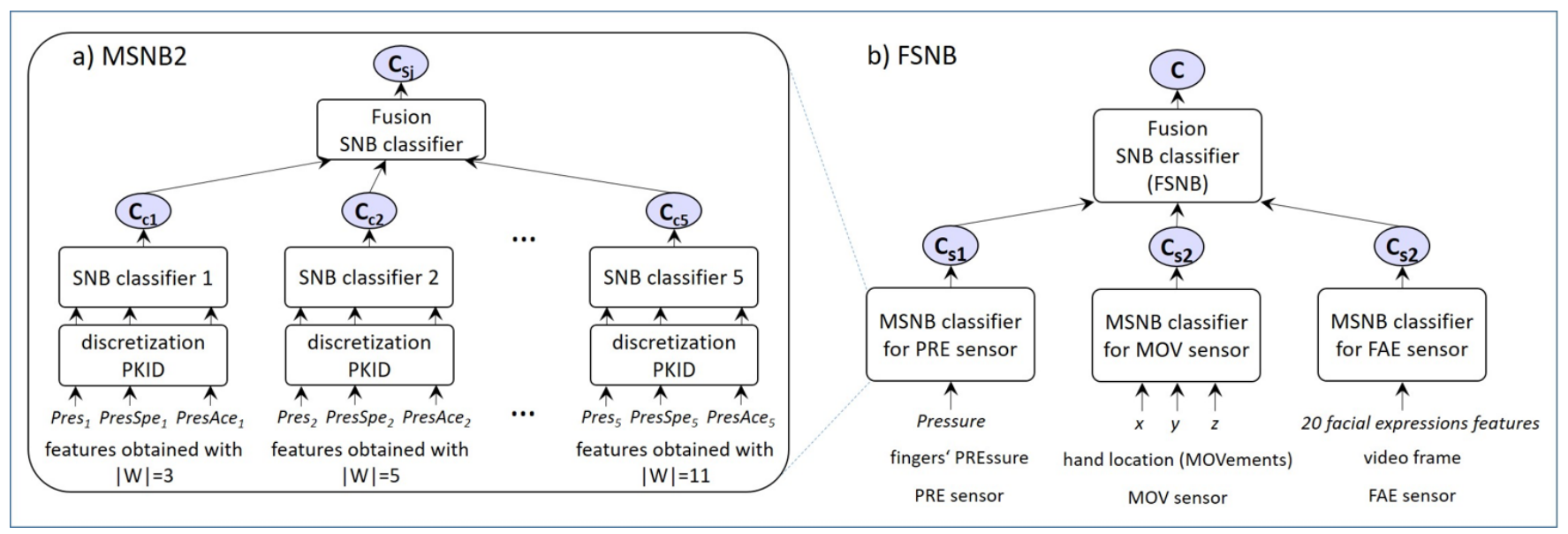

Figure 6: MSNB2 and FSNB classifiers. a) MSNB2 classifier shifts a set of parallel windows $W$ of different odd sizes, $|W|=3,5,7,9,11$; all concurrently centred around the same time sample of the respective sensor. b) FSNB classifier which contains a MSNB classifier for each sensor. Acronyms meanings: $C, C_{c k}, C_{s j}=$ class of the respective classifier for the same affective state, e.g. anxiety; Pres $=$ pressure, PresSpe $=$ pressure speed and PresAce $=$ pressure acceleration .

Table 1: Performance comparisons between PRE, MOV, FAE, and the fusion PRE-MOV-FAE for each state using AUC average results across the $\mathbf{1 0}$ folds and across the five patients (mean \pm std.deviation). The best results for each state are highlighted in bold type.

\begin{tabular}{|c|c|c|c|c|}
\hline Sensor & Tiredness & Anxiety & Pain & Motivation \\
\hline \multicolumn{5}{|c|}{ classifier: MSNB2 } \\
\hline PRE & $0.830 \pm 0.129$ & $0.826 \pm 0.109$ & $0.705 \pm 0.111$ & $0.829 \pm 0.121$ \\
\hline MOV & $0.874 \pm 0.101$ & $0.841 \pm 0.069$ & $0.763 \pm 0.100$ & $0.834 \pm 0.090$ \\
\hline FAE & $0.927 \pm 0.081$ & $0.941 \pm 0.061$ & $0.916 \pm 0.118$ & $0.922 \pm 0.091$ \\
\hline \multicolumn{5}{|l|}{ classifier: FSNB } \\
\hline PRE-MOV-FAE & $0.957 \pm 0.050$ & $0.952 \pm 0.053$ & $0.943 \pm 0.067$ & $0.932 \pm 0.076$ \\
\hline
\end{tabular}

the states. Although the facial expressions sensor (FAE) achieved the best classification scores among the three available sensors; the face detection in the video stream was sometimes lost and the multimodal system only had the sensors of hand movements (MOV) and fingers' pressure (PRE) which had classification scores around $80 \%$. For this reason, the PRE and MOV sensors are useful to contribute to sustain the system when the FAE sensor is absent.

\section{CONCLUSIONS AND FUTURE WORK}

The problem of a multimodal model which include finger's pressure, hand movements and facial expressions sensor of patients' upper limb motions and the patients' facial expressions respectively, has been explored for contributing to recognize affective states in a virtual rehabilitation platform. The performance of the chosen strategy achieved results above 93 percent. FAE sensor had the best results among the three sensors, but the other sensors are useful because their obtained results over $70 \%$ and they were present when the FAE sensor failed to detect the face in the video frames.

Our proposal only includes visual (facial expressions) and body movements (hand movements and fingers' pressure) modalities,

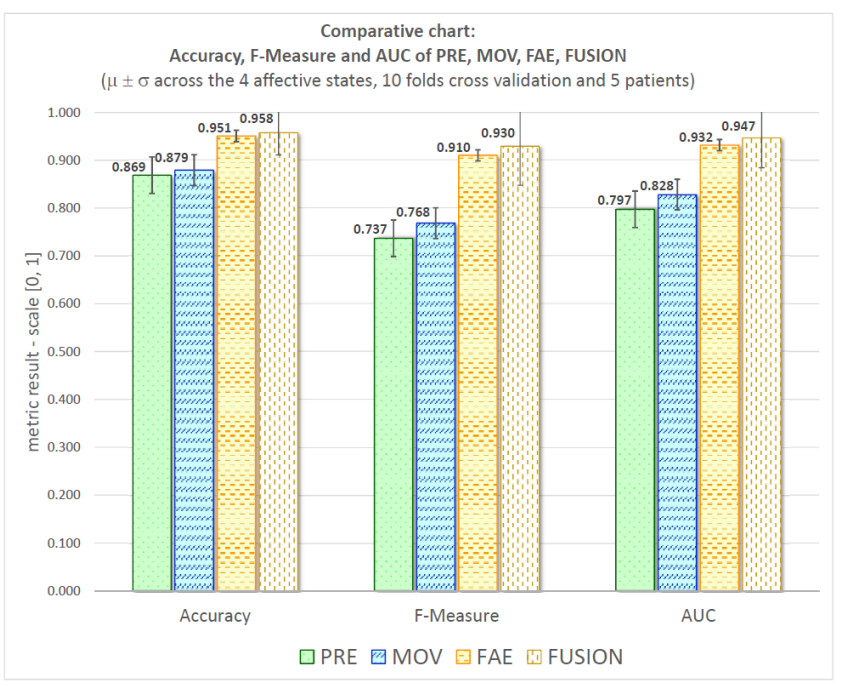

Figure 7: Comparative chart of the Accuracy, F-Measure, and AUC of PRE, MOV, and FAE sensors, and the Fusion results.

but it can be joined on other modalities as eye gaze (noninvasive sensor) and some physiological signals (Galvanic Skin Response, Electrocardiography, Respiration, and EEG) in future works.

\section{ACKNOWLEDGMENTS}

blinded.

\section{REFERENCES}

[1] Themis Balomenos, Amaryllis Raouzaiou, Spiros Ioannou, Athanasios Drosopoulos, Kostas Karpouzis, and Stefanos Kollias. 2004. Emotion analysis in manmachine interaction systems. In Proceedings of the International Workshop on Machine Learning for Multimodal Interaction. Springer, 318-328.

[2] Andrea Bandini, Silvia Orlandi, Hugo Jair Escalante, Fabio Giovannelli, Massimo Cincotta, Carlos A Reyes-Garcia, Paola Vanni, Gaetano Zaccara, and Claudia. 
Manfredi. 2017. Analysis of facial expressions in parkinson's disease through video-based automatic methods. Fournal of neuroscience methods 281 (2017), $7-20$.

[3] Andrea Bonarini, Maurizio Garbarino, Matteo Matteucci, and Simone Tognetti. 2010. Affective evaluation of robotic rehabilitation of upper limbs in post-stroke subjects. In Proceedings of the 1st Workshop on the Life Sciences at Politecnico di Milano. 290-293.

[4] CK Chow and CN Liu. 1968. Approximating discrete probability distributions with dependence trees. IEEE Transactions on Information Theory 14, 3 (1968), 462-467.

[5] Sidney K D'mello and Jacqueline Kory. 2015. A review and meta-analysis of multimodal affect detection systems. ACM Computing Surveys (CSUR) 47, 3 (2015), 43:1-43:36.

[6] Richard O Duda, Peter E Hart, et al. 1973. Pattern classification and scene analysis. Vol. 3. Wiley New York.

[7] Panagiotis P Filntisis, Niki Efthymiou, Petros Koutras, Gerasimos Potamianos, and Petros Maragos. 2019. Fusing Body Posture with Facial Expressions for Joint Recognition of Affect in Child-Robot Interaction. arXiv preprint arXiv:1901.01805 (2019).

[8] Hatice Gunes and Massimo Piccardi. 2007. Bi-modal emotion recognition from expressive face and body gestures. Journal of Network and Computer Applications 30, 4 (2007), 1334-1345.

[9] Hatice Gunes and Massimo Piccardi. 2009. Automatic temporal segment detection and affect recognition from face and body display. IEEE Transactions on Systems, Man, and Cybernetics, Part B (Cybernetics) 39, 1 (2009), 64-84.

[10] Patricia Kan, Rajibul Huq, Jesse Hoey, Robby Goetschalckx, and Alex Mihailidis 2011. The development of an adaptive upper-limb stroke rehabilitation robotic system. Fournal of Neuroengineering and Rehabilitation 8, 1 (2011), 1-18.

[11] Miriam Martínez Arrollo. 2007. Aprendizaje de clasificadores bayesianos estáticos y dinámicos. Ph.D. Dissertation. Instituto Tecnológico y de Estudios Superiores de Monterrey, México.

[12] Miriam Martínez-Arroyo and L Enrique Sucar. 2006. Learning an optimal naive Bayes classifier. In Proceedings of the 18th International Conference on Pattern
Recognition, 2006. (ICPR 2006), Vol. 3. IEEE, 1236-1239.

[13] Michael J Pazzani. 1996. Searching for dependencies in Bayesian classifiers. In Learning from Data. Springer, 239-248.

[14] Soujanya Poria, Erik Cambria, Rajiv Bajpai, and Amir Hussain. 2017. A review of affective computing: From unimodal analysis to multimodal fusion. Information Fusion 37 (2017), 98-125.

[15] Jesús Joel Rivas. 2015. Clasificador Semi-Nä̈ve Bayes con multiresolución para la estimación de estados afectivos: Aplicación en Rehabilitación Virtual. Master's thesis. Instituto Nacional de Astrofísica, Óptica y Electrónica (INAOE), Puebla, Mexico.

16] Jesús Joel Rivas, Felipe Orihuela-Espina, Lorena Palafox, Nadia Berthouze, María del Carmen Lara, Jorge Hernández-Franco, and Enrique Sucar. 2018. Unobtrusive inference of affective states in virtual rehabilitation from upper limb motions: A feasibility study. IEEE Transactions on Affective Computing (2018). https: //doi.org/10.1109/TAFFC.2018.2808295

[17] Jesús J Rivas, Felipe Orihuela-Espina, L Enrique Sucar, Lorena Palafox, Jorge Hernández-Franco, and Nadia Bianchi-Berthouze. 2015. Detecting affective states in virtual rehabilitation. In Proceedings of the 9th International Conference on Pervasive Computing Technologies for Healthcare (PervasiveHealth 2015). IEEE, 287-292.

[18] Mohammad Soleymani, Jeroen Lichtenauer, Thierry Pun, and Maja Pantic. 2012. A multimodal database for affect recognition and implicit tagging. IEEE Transactions on Affective Computing 3, 1 (2012), 42-55.

[19] Luis Enrique Sucar, Ron Leder, Jorge Hernández, Israel Sánchez, and Gildardo Azcárate. 2009. Clinical evaluation of a low-cost alternative for stroke rehabilitation. In Proceedings of the 11th International Conference on Rehabilitation Robotics (ICORR 2009). IEEE, 863-866.

[20] Luis Enrique Sucar, Felipe Orihuela-Espina, Roger Luis Velazquez, David J Reinkensmeyer, Ronald Leder, and Jorge Hernández-Franco. 2014. Gesture therapy: An upper limb virtual reality-based motor rehabilitation platform. IEEE Transactions on Neural Systems and Rehabilitation Engineering 22, 3 (2014), 634643 